\title{
NUCLEAR TOPOLOGIES CONSISTENT WITH \\ A DUALITY
}

MICHAEL B. DOLLINGER

Let $E$ be a (Hausdorff) locally convex space, and let $E^{\prime}$ be its dual space. A subset $B \subset E^{\prime}$ is said to be prenuclear if there exist a $\sigma\left(E^{\prime}, E\right)$ closed equicontinuous subset $A \subset E^{\prime}$ and a positive Radon measure $\mu$ on $A$ such that for each $x \in E$,

$$
\sup _{y^{\prime} \in B}\left|\left\langle x, y^{\prime}\right\rangle\right| \leqq \int_{A}\left|\left\langle x, x^{\prime}\right\rangle\right| d \mu\left(x^{\prime}\right) .
$$

Pietsch has shown that $E$ is a nuclear space if and only if every equicontinuous subset of $E^{\prime}$ is prenuclear (see [1] or [3]). We shall use this result to characterize all nuclear topologies on a locally convex space which are consistent with a given duality. We refer the reader to [3] for the basic results and notation that we shall use.

We begin with a definition.

Definition. Let $E$ be a locally convex space, and let $B_{0} \subset E^{\prime}$. We shall say that $B_{0}$ is a hypernuclear set if there exists a sequence $\left\{\left(B_{n}, \mu_{n}\right): n=1,2, \ldots\right\}$ where each $B_{n}$ is a $\sigma\left(E^{\prime}, E\right)$-closed equicontinuous subset of $E^{\prime}$ and $\mu_{n}$ is a positive $\sigma\left(E^{\prime}, E\right)$-Radon measure on $B_{n}$ such that for each $n \geqq 1$ and each $x \in E$,

$$
\sup _{\nu^{\prime} \in B_{n-1}}\left|\left\langle x, y^{\prime}\right\rangle\right| \leqq \int_{B_{n}}\left|\left\langle x, x^{\prime}\right\rangle\right| d \mu_{n}\left(x^{\prime}\right) .
$$

We shall say that the sequence $\left\{\left(B_{n}, \mu_{n}\right): n=1,2, \cdots\right\}$ corresponds to the hypernuclear set $B_{0}$.

In the following proposition, we state some elementary properties of hypernuclear sets. The proof is fairly direct, and so it has been omitted.

Proposition 1. (a) Finite subsets of $E^{\prime}$ are hypernuclear.

(b) If $A$ and $B$ are hypernuclear and $\lambda$ is a scalar, then $A \cup \lambda B$ is hypernuclear.

(c) If $A$ is hypernuclear and $B \subset A$, then $B$ is hypernuclear.

(d) If $A$ is hypernuclear, then the $\sigma\left(E^{\prime}, E\right)$-closed convex circled hull of $A$ is hypernuclear.

(e) If $A$ is hypernuclear, then $A$ is prenuclear and hence equicontinuous.

Received by the editors March 13, 1969. 
(f) If $B_{0}$ is hypernuclear and $\left\{\left(B_{n}, \mu_{n}\right): n=1,2 \ldots\right\}$ is a corresponding sequence, then each $B_{n}$ is also hypernuclear.

We remark here that the hypernuclear subsets of $E^{\prime}$ depend on the particular topology on $E$. Moreover, it follows from the theorem of Pietsch stated above that $E$ is a nuclear space if and only if every equicontinuous subset of $E^{\prime}$ is hypernuclear.

Definition. Let $E$ be a locally convex space. A family $\mathcal{F}$ of hypernuclear subsets of $E^{\prime}$ is said to be a full family if the following conditions hold.

(a) $\bigcup \mathfrak{F}=E^{\prime}$.

(b) If $A, B \in \mathcal{F}$, then $A \cup \lambda B \in \mathcal{F}$ for all scalars $\lambda$.

(c) If $A \in \mathcal{F}$ and $B \subset A$, then $B \in \mathcal{F}$.

(d) If $A \in \mathcal{F}$, then the $\sigma\left(E^{\prime}, E\right)$-closed convex circled hull of $A$ belongs to $\mathcal{F}$.

(e) If $B_{0} \in \mathcal{F}$, then there is at least one corresponding sequence $\left\{\left(B_{n}, \mu_{n}\right): n=1,2, \cdots\right\}$ such that $B_{n} \in \mathcal{F}$ for all $n$.

In the theorems below, it will be important to distinguish among different topologies on the same space. By $(E, \rho)$, we shall mean that the space $E$ is being considered with the topology $\rho$.

TheOREm 2. Let $(E, \rho)$ be a locally convex space, and let $\mathfrak{F}$ be a full family of $\rho$-hypernuclear subsets of $E^{\prime}$. Let $\rho_{\mathcal{F}}$ be the topology on $E$ of uniform convergence on the members of $\mathfrak{F}$. Then $\left(E, \rho_{\mathfrak{F}}\right)$ is a nuclear space having the same dual space as $(E, \rho)$.

Proof. Since members of $\mathfrak{F}$ are all $\rho$-equicontinuous, we conclude that $\left(E, \rho_{\mathcal{F}}\right)^{\prime}=(E, \rho)^{\prime}$. The $\rho_{\mathcal{F}}$-equicontinuous subsets of $E^{\prime}$ are just the members of $\mathcal{F}$, and so it suffices, by Pietsch's result, to show that each $B_{0} \in \mathcal{F}$ is $\rho_{\mathfrak{F}}$-prenuclear. Let $\left\{\left(B_{n}, \mu_{n}\right): n=1,2, \cdots\right\}$ be a $\rho$-corresponding sequence for $B_{0}$ such that $B_{n} \in \mathcal{F}$ for all $n$. Now $\mu_{1}$ is a $\sigma\left(\left(E, \rho_{\mathfrak{F}}\right)^{\prime}, E\right)$-Radon measure on the $\rho_{\mathfrak{F}}$-equicontinuous set $B_{1}$, and hence $B_{0}$ is also $\rho_{\mathfrak{F}}$-prenuclear.

In the following theorem, $\tau(E, F)$ will denote the Mackey topology on $E$, that is, the largest topology on $E$ which is consistent with the duality $\langle E, F\rangle$.

Theorem 3. Let $\langle E, F\rangle$ be a duality. A topology $\rho$ on $E$ is consistent with the duality and makes $E$ a nuclear space if and only if it is the topology of uniform convergence on some full family of $\tau(E, F)$-hypernuclear sets in $F$.

Proof. The "if" part is simply the previous theorem with $\rho$ $=\tau(E, F)$. 
Let $\rho$ be a topology on $E$ such that $(E, \rho)$ is a nuclear space and $(E, \rho)^{\prime}=F$. Then every member of the family $\mathcal{F}$ of all $\rho$-equicontinuous subsets of $F$ is $\rho$-hypernuclear. We claim that $\mathcal{F}$ is a full family of $\tau(E, F)$-hypernuclear sets. Conditions (a)-(d) for $\mathcal{F}$ are easily checked. Every $\rho$-equicontinuous set is $\tau(E, F)$-equicontinuous; and hence it follows that if $B_{0} \in \mathcal{F}$, then any $\rho$-corresponding sequence $\left\{\left(B_{n}, \mu_{n}\right): n=1,2, \cdots\right\}$ will also be $\tau(E, F)$-corresponding. Thus $\mathfrak{F}$ is also a full family of $\tau(E, F)$-hypernuclear sets.

It follows from the above results that the weak topology and the topology of uniform convergence on all $\tau(E, F)$-hypernuclear sets are respectively the weakest and strongest topologies on $E$ which are consistent with the duality $\langle E, F\rangle$ and which make $E$ a nuclear space.

We shall conclude with an example of a prenuclear set which is not hypernuclear.

EXAMPLE. Let $I$ be an uncountable index set. Let $l_{1}(I)$ and $l_{2}(I)$ be the collections of all families $\left\{x_{\alpha}: \alpha \in I\right\}$ of scalars such that $\sum_{I}\left|x_{\alpha}\right|<\infty$ and $\sum_{I}\left|x_{\alpha}\right|^{2}<\infty$, respectively. These spaces become Banach spaces under the norms $\left\|\left\{x_{\alpha}\right\}\right\|_{1}=\sum_{I}\left|x_{\alpha}\right|$ and $\left\|\left\{x_{\alpha}\right\}\right\|_{2}$ $=\left(\sum_{I}\left|x_{\alpha}\right|^{2}\right)^{1 / 2}$.

The identity map $i: l_{1}(I) \rightarrow l_{2}(I)$ is absolutely summing $[2$, p. 39]; or equivalently $\left[2\right.$, p. 36], there is a positive $\sigma\left(l_{1}(I)^{\prime}, l_{1}(I)\right)$-Radon measure $\mu$ on the unit ball $M$ of $l_{1}(I)^{\prime}$ such that for each $x \in l_{1}(I)$

$$
\|x\|_{2} \leqq \int_{M}\left|\left\langle x, x^{\prime}\right\rangle\right| d \mu\left(x^{\prime}\right) .
$$

Let $B$ be the unit ball of $l_{2}(I)$, and consider $B$ as a subset of $l_{1}(I)^{\prime}$. Then for $y \in B$ and $x \in l_{1}(I)$,

$$
\begin{aligned}
|\langle x, y\rangle| & \leqq\|x\|_{2}\|y\|_{2} \leqq\|x\|_{2} \\
& \leqq \int_{M}\left|\left\langle x, x^{\prime}\right\rangle\right| d \mu\left(x^{\prime}\right) .
\end{aligned}
$$

Hence $B$ is a prenuclear subset of $l_{1}(I)^{\prime}$.

Now assume that $B$ is a hypernuclear subset of $l_{1}(I)^{\prime}$, and let $\rho$ be the topology on $l_{1}(I)$ of uniform convergence on all hypernuclear subsets of $l_{1}(I)^{\prime}$. Both of the injection maps $j$ and $k$

$$
\left(l_{1}(I),\|-\|_{1}\right) \stackrel{j}{\rightarrow}\left(l_{1}(I), \rho\right) \stackrel{k}{\rightarrow}\left(l_{2}(I),\|-\|_{2}\right)
$$

can be seen to be continuous. By Theorem $2,\left(l_{1}(I), \rho\right)$ is a nuclear space, and hence $k$ is a nuclear map. Since the composition of a nu- 
clear map with a continuous map is still nuclear, we conclude that $i=k \circ j$ is a nuclear map. But this is a contradiction since $i$ is not even compact $[2$, p. 40$]$. Hence $B$ cannot be hypernuclear.

\section{REFERENCES}

1. A. Pietsch, Eine neue Charakterisierung der nuklearen lokalconvexen Räume. I, Math. Nachr. 25 (1963), 31-36.

2. - Nukleare lokalconvexe Räume, Akademie-Verlag, Berlin, 1965.

3. H. H. Schaefer, Topological vector spaces, Macmillan, New York, 1966.

Louisiana State University, Baton Rouge 